\title{
A full Nesterov-Todd step primal-dual path-following interior-point algorithm for semidefinite linear complementarity problems
}

\author{
Mohamed Achache ${ }^{1, *}$ and Nesrine Tabchouche ${ }^{1}$ \\ ${ }^{1}$ Laboratoire de Mathématiques Fondamentales et Numériques, Sétif1, Sétif, 19000, Algérie \\ E-mail: 〈achache_m@univ-setif.dz, tabchouche_nesrine@yahoo.fr 〉
}

\begin{abstract}
In this paper, a feasible primal-dual path-following interior-point algorithm for monotone semidefinite linear complementarity problems is proposed. At each iteration, the algorithm uses only full Nesterov-Todd feasible steps for tracing approximately the central-path and getting an approximated solution of this problem. Under a new appropriate choices of the threshold $\tau$ which defines the size of the neighborhood of the central-path and of the update barrier parameter $\theta$, we show that the algorithm is well-defined and enjoys the locally quadratically convergence. Moreover, we prove that the short-step algorithm deserves the best known iteration bound, namely, $O\left(\sqrt{n} \log \frac{n}{\epsilon}\right)$. Finally, some numerical results are reported to show the practical performance of the algorithm.
\end{abstract}

Keywords: Semidefinite linear complementarity; Interior-point algorithm; Short-step method; Polynomial complexity.

Received: October 10, 2017; accepted: July 18, 2018; available online: July 24, 2018

DOI: 10.17535 /crorr.2018.0004

\section{Introduction}

Let $\mathbb{S}^{n}$ denotes the linear space of all $n \times n$ real symmetric matrices and $\mathbb{S}_{+}^{n} \subset \mathbb{S}^{n}$ be the closed convex cone of symmetric positive semidefinite matrices. Given a linear transformation $\mathcal{L}: \mathbb{S}^{n} \rightarrow \mathbb{S}^{n}$ and a symmetric matrix $Q \in \mathbb{S}^{n}$, the semidefinite linear complementarity problem (SDLCP), is the problem of finding a pair of matrices $(X, Y) \in \mathbb{S}^{n} \times \mathbb{S}^{n}$ such that

$$
X, Y \in \mathbb{S}_{+}^{n}, \quad Y=\mathcal{L}(X)+Q, X \bullet Y=0,
$$

where $X \bullet Y$ denotes the trace of the matrix product $X Y$.

The SDLCPs is an important class of mathematical programming which finds many applications in control theory, linear and bilinear inequalities and other optimization related problems. It includes also as a special, the geometric SDLCP [13], the standard linear complementarity problem (LCP) [5], the primal-dual pair of linear (LO), convex quadratic (CQO) and semidefinite optimization $(\mathrm{SDO})$. In references $[4,9]$, the reader will find the state of art of SDLCPS and its applications.

There are many approaches for solving the class of monotone SDLCP. Among them primaldual path-following interior-point methods (IPMs) gained much more attention than others. Their derived algorithms enjoy polynomial complexity and they are highly efficient in practice [17]. Thus motivates researchers to extend it to other convex optimization problems and mathematical programming such as $\mathrm{CQO}$, SDO, convex quadratic semidefinite optimization

${ }^{*}$ Corresponding author. 
(CQSDO), LCP, SDLCP and so on. For an overview of these methods, we refer to the references $[1,3,4,5,11,12,14,15,17,18]$.

For instance, there is a considerable variant of primal-dual IPMs for solving SDLCPs. Kojima et al. [13], first proved the existence and the uniqueness of the central-path of SDLCP and then presented an interior-point method to solve it. Their search directions is based on Nesterov-Todd scheme. Also Tseng [17], proposed an infeasible interior-point method for solving this problem. Later on, Achache and Boudiaf [2], study the complexity of a damped Newton Nesterov-Todd step primal-dual IPMs for SDLCPs. They proved its complexity for large and short-step methods. Besides, Wang and Bai [18], suggested a short-step primal-dual interiorpoint algorithm for solving the SDO problems. Their search directions is based on the technique of an algebraically equivalent transformation applied to the nonlinear equations from the system of equations which defines the central-path. They also computed its complexity. Their work is an extension of Darvay's work for LO [7]. Chen [6], presented a primal-dual IPMs for geometric SDLCP. His search directions is based on the M-Z family. He also proved its polynomial convergence. Recently, Achache and Guerra [2], designed a feasible short full-Nesterov step algorithm for solving CQSDOs. They proved that the iteration bound is $O\left(\sqrt{n} \log \frac{n}{\epsilon}\right)$. Their analysis was inspired from the work of De Kelerk [8], for SDO. Furthermore, Kheirfam and Haghighi [12], presented a full-Newton step feasible IPMs for solving the $P_{*}(\kappa)$-LCP linear complementarity problem based on a new search direction. They derive the iteration bound for their algorithm, which coincides with the best-known iteration bound for these types of algorithms. Very recently, Mansouri et al. [15], proposed a feasible IPMs for solving SDLCPs based on a classical kernel function and the Nesterov-Todd search directions. They proved that their short-step algorithm will terminates after at most $O\left(\sqrt{n} \log \frac{n}{\epsilon}\right)$ iterations.

The majority of the above mentioned short-step primal-dual path-following IPMs are based on tracing approximately the central-path by restricting the given strictly feasible iterates in a neighborhood of it and decreasing the duality gap to zero for reaching an optimal solution for this problem using defaults of the threshold $\tau$ which defines the size of this neighborhood and of the update barrier parameter $\theta$. These defaults play an important role in the analysis and the practical performance of these algorithms.

In this paper, our motivation is to offer on one hand new defaults for these parameters and on the other hand we elaborate across this latter a feasible primal-dual path-following short-step interior-point algorithm for solving (1). We show under these new defaults that the algorithm is well-defined and enjoys the locally quadratically convergence. Moreover, we prove that the short-step algorithm deserves the best known iteration bound, namely, $O\left(\sqrt{n} \log \frac{n}{\epsilon}\right)$. This iteration bound is as good as the bound for LO [7, 16], CQO [1], SDO [8, 18], CQSDO [2], $P_{*}(\kappa)$-LCP [12] if $\kappa=0$, and SDLCP [15], cases. Here, we reconsider the analysis used in [2] and [8] and we make it suited for our case. Finally, some numerical results on two problems constructed by Lyapunov and two-sided linear transformations show that the new proposed defaults are well promising and perform well enough in practice in comparison with some other existing defaults in the literature.

The rest of the paper is built as follows. In section 2, the generic full-Newton step feasible IPMs for SDLCP is presented. In section 3, detailed proofs of the complexity of the proposed algorithm are given. Section 4, provides some numerical results while some conclusions and remarks are drawn in section 5 .

The following notations are used throughout the paper. $\mathbb{R}^{n}$ denotes the $n$-dimensional euclidean space. The set of all $n \times n$ squared matrices is denoted by $\mathbb{R}^{n \times n}$. The trace of a matrix $A=\left(a_{i j}\right)$ is denoted by $\operatorname{Tr}(A)$ and is defined by $\operatorname{Tr}(A)=\sum_{i=1}^{n} a_{i i}$. The Frobenius and the spectral norms of a matrix $A$ in $\mathbb{R}^{n \times n}$ are defined by $\|A\|_{F}=\sqrt{A \bullet A}=\sqrt{\operatorname{Tr}\left(A A^{T}\right)}$ and $\|A\|$, respectively. If $X \in \mathbb{S}^{n}$ is positive semidefinite (definite), we write $X \succeq 0(X \succ 0)$ ). For any squared matrix $V$, det $V$ denotes its determinant, meanwhile, $\lambda_{\min }(V)$ and $\lambda_{\max }(V)$ denote, respectively, its smallest and its largest eigenvalue. The symmetric positive definite square root 
of any matrix $X \succ 0$ is denoted by $X^{1 / 2}$. The notation $A \sim B \Leftrightarrow A=P B P^{-1}$ means that $A$ is similar to $B$. If $f(x) \geq 0$, is a real valued function of a real nonnegative variable, then $f(x)=O(x)$ means that $f(x) \leq c x$ for some constant $c$. Finally, the identity matrix of order $n$ and the vector of ones are denoted by $I$ and $e$, respectively.

\section{A full Nesterov-Todd step feasible IPM}

In this section, we first recall the notion of the central-path for SDLCP. Then we derive the Nesterov-Todd search directions for SDLCP. Finally, the generic full-Newton step feasible interior-point algorithm based on the Nesterov-Todd search directions is presented.

\subsection{The central-path for SDLCP}

Throughout the paper, we assume that the SDLCP (1) satisfies the following conditions.

- Interior-Point-Condition (IPC). There exists a pair of matrices $\left(X^{0}, Y^{0}\right)$ such that:

$$
Y^{0}-\mathcal{L}\left(X^{0}\right)=Q, X^{0} \succ 0, Y^{0} \succ 0 .
$$

- Monotonicity. The symmetric linear transformation $\mathcal{L}$ satisfies:

$$
\mathcal{L}(X) \bullet X \geq 0, \quad \forall X \in \mathbb{S}^{n} .
$$

Since for $X, Y \in \mathbb{S}_{+}^{n}$, we have $X \bullet Y=0$ if and only if $X Y=0$. Then, finding a solution of (1) is equivalent to solving the following system:

$$
\left\{\begin{array}{l}
Y-\mathcal{L}(X)=Q \\
X Y=0, \quad X, Y \succeq 0
\end{array}\right.
$$

The basic idea of primal-dual IPMs is to replace the second equation in (2) by the parameterized equation $X Y=\mu I, \mu>0$. Hence, the system (2) becomes

$$
\left\{\begin{array}{l}
Y-\mathcal{L}(X)=Q \\
X Y=\mu I, \quad X, Y \succeq 0 .
\end{array}\right.
$$

If IPC holds then for each $\mu>0$, system (3) has a unique solution $(X(\mu), Y(\mu))$ which is called the $\mu$-centers of SDLCP. The set of the $\mu$-centers gives a homotopy path which is called the central-path of SDLCP. If $\mu \mapsto 0$, then the limit of the central-path exists, and since the limit points satisfy the complementarity condition i.e., $X Y=0$, the limit yields a solution for SDLCP. Detailed proofs of the existence and uniqueness of the central-path can be found in Kojima et al., [13].

\subsection{The Nesterov-Todd search directions}

Applying Newton's method to system (3), we get the following system

$$
\left\{\begin{array}{l}
\Delta Y=\mathcal{L}(\Delta X) \\
\Delta X Y+X \Delta Y=\mu I-X Y
\end{array}\right.
$$

or equivalently

$$
\left\{\begin{array}{l}
\Delta Y=\mathcal{L}(\Delta X) \\
\Delta X+X \Delta Y Y^{-1}=\mu Y^{-1}-X
\end{array}\right.
$$


Clearly, $\Delta X$ is not symmetric due to the matrix $X \Delta Y Y^{-1}$. Therefore, a way to symmetrizing the second equation in (5) is to introduce an invertible matrix $P$ and to replace it by the equation $\Delta X+P \Delta Y P^{T}=\mu Y^{-1}-X$, such that the resulting new system has a unique symmetric solution. Thus, we obtain

$$
\left\{\begin{array}{l}
\Delta Y=\mathcal{L}(\Delta X) \\
\Delta X+P \Delta Y P^{T}=\mu Y^{-1}-X
\end{array}\right.
$$

In this paper, we consider the symmetrization scheme of Nesterov-Todd [14]. So we use

$$
P=X^{1 / 2}\left(X^{1 / 2} Y X^{1 / 2}\right)^{-1 / 2} X^{\frac{1}{2}}=Y^{-1 / 2}\left(Y^{1 / 2} X Y^{1 / 2}\right)^{1 / 2} Y^{-1 / 2} .
$$

To simplify the matters, we define $D=P^{1 / 2}$. The symmetric matrix $D$ can be used to rescale $X$ and $Y$ to the same matrix $V[8]$, defined by

$$
V:=\frac{1}{\sqrt{\mu}} D^{-1} X D^{-1}=\frac{1}{\sqrt{\mu}} D Y D .
$$

Due to (7), it easy to see that $V^{2}=\frac{1}{\mu} D^{-1} X Y D$, i.e., $V^{2} \sim \frac{1}{\mu} X Y$. Moreover, let us further define the following notations

$$
D_{X}=\frac{1}{\sqrt{\mu}} D^{-1} \Delta X D^{-1}, D_{Y}=\frac{1}{\sqrt{\mu}} D \Delta Y D .
$$

Again due to (7) and (8), system (6) can be rewritten as follows:

$$
\left\{\begin{array}{l}
\overline{\mathcal{L}}\left(D_{X}\right)=D_{Y} \\
D_{X}+D_{Y}=P_{V}
\end{array}\right.
$$

where $\overline{\mathcal{L}}\left(D_{X}\right)=D L\left(D D_{X} D\right) D$ and

$$
P_{V}=V^{-1}-V
$$

Because $\mathcal{L}$ is monotone, then by considering the first equation in (6), and due (8), the scaled directions satisfy the non-orthogonality property since

$$
D_{X} \bullet D_{Y}=\frac{1}{\mu} \Delta X \bullet \Delta Y=\frac{1}{\mu} \Delta X \bullet \mathcal{L}(\Delta X) \geq 0 .
$$

Note that for SDO case $D_{X} \bullet D_{Y}=0$. It is the only difference between SDO problem and SDLCP problem. Thus makes the analysis different.

The search direction $D_{X}$ and $D_{Y}$ are obtained by solving (9) so that $\Delta X$ and $\Delta Y$ are computed via (8). Then the new iterate is obtained by taking a full NT-step as follows:

$$
X_{+}:=X+\Delta X, Y_{+}:=Y+\Delta Y
$$

For the analysis of the algorithm, we define a norm-based proximity measure as follows:

$$
\delta(X, Y ; \mu):=\delta(V)=\frac{1}{2}\left\|P_{V}\right\|_{F} .
$$

Clearly,

$$
\delta(V)=0 \Leftrightarrow V=I \Leftrightarrow X Y=\mu I .
$$

Hence, the value of $\delta(V)$ can be considered as a measure for the distance between the matrix pair of matrices $(X, Y)$ and the corresponding $\mu$-center $(X(\mu), Y(\mu))$. 


\subsection{The generic interior-point algorithm}

In this subsection, the general outline of the generic interior-point primal-dual algorithm for SDLCP is described as follows. First, we use a suitable threshold (default) value $\tau>0$, with $0<\tau<1$ and we suppose that a strictly feasible initial point $\left(X^{0}, Y^{0}\right)$ exists such that $\delta\left(X^{0}, Y^{0} ; \mu_{0}\right) \leq \tau$, for certain $\mu_{0}$ is known. Using the obtained search directions $(\Delta X, \Delta Y)$ and taking a full-Newton step, the algorithm produces a new iterate $\left(X_{+}, Y_{+}\right)=(X+\Delta X, Y+\Delta Y)$. Then, it updates the barrier parameter $\mu$ to $(1-\theta) \mu$ with $0<\theta<1$ and solve the Newton system and then target a new $\mu$-center and so on. This procedure is repeated until the stopping criterion $n \mu \leq \epsilon$ is satisfied for a given accuracy parameter $\epsilon$. The details of our generic full NT-step feasible interior-point algorithm for SDLCP is now presented in Fig.1. as follows.

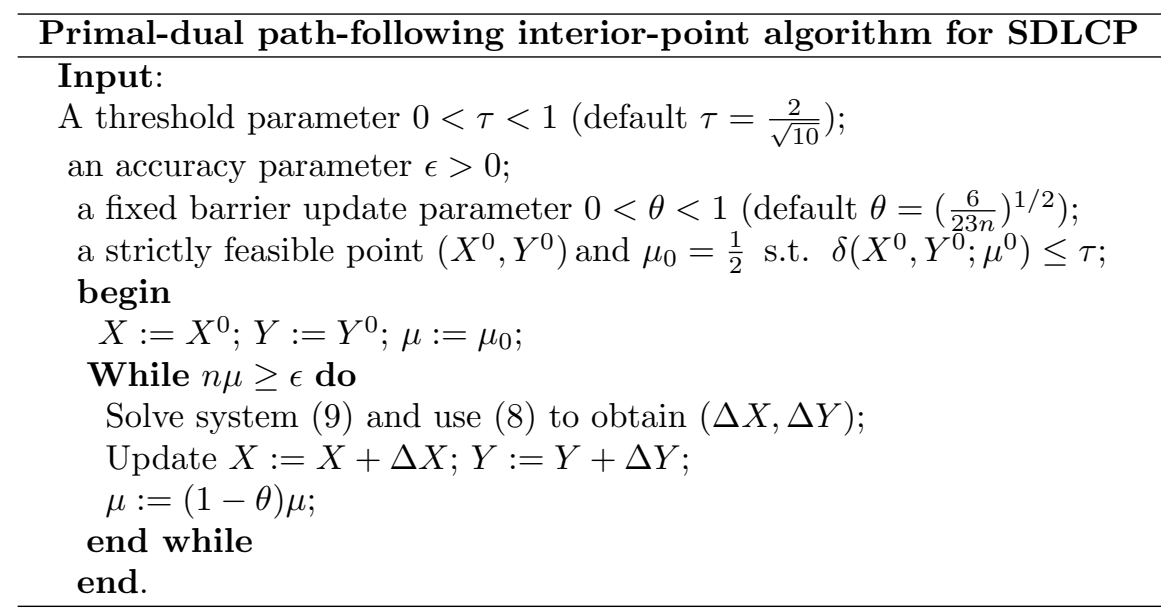

Figure 1: Algorithm 2.3

\section{Complexity analysis}

In this section we will show that Algorithm 2.3 can solve the SDLCPs in polynomial-time.

\subsection{Feasibility and locally quadratically convergence of the feasible NT-step}

In this subsection, we first investigate the strict feasibility of the full NT-step. Then, we mainly prove the locally quadratically convergent of full NT-step to the target point $(X(\mu), Y(\mu))$.

For the analysis of the algorithm, we consider the $n \times n$ real symmetric matrix $D_{X Y}$ given by

$$
D_{X Y}:=\frac{1}{2}\left[D_{X} D_{Y}+D_{Y} D_{X}\right] .
$$

We recall some useful lemmas in $[2,8]$, which will be used in the proof of Lemma 5 .

Let $0 \leq \alpha \leq 1$, we define

$$
X(\alpha):=X+\alpha \Delta X, Y(\alpha):=Y+\alpha \Delta Y .
$$

Lemma 1 (Lemma 6.1 in [8]). Suppose that $X \succ 0$ and $Y \succ 0$. If

$$
\operatorname{det}(X(\alpha) Y(\alpha))>0, \forall 0 \leq \alpha \leq \bar{\alpha},
$$

then $X(\bar{\alpha}) \succ 0$ and $Y(\bar{\alpha}) \succ 0$. 
Lemma 2 (Lemma A.1 in [8]). Let $Q \succ 0$, and let $M \in \mathbb{R}^{n \times n}$ be skew-symmetric. One has $\operatorname{det}(Q+M)>0$ if $Q \succ 0$. Moreover, if $\lambda_{i}(Q+M) \in \mathbb{R}$ for $i=1, \ldots, n$, then $0<\lambda_{\min }(Q) \leq$ $\lambda_{\min }(Q+M) \leq \lambda_{\max }(Q+M) \leq \lambda_{\max }(Q)$, which implies $(Q+M) \succ 0$.

Lemma 3 (Lemma 3.3 in [2]). Let $\delta:=\delta(X, Y ; \mu)$ and $\left(D_{X}, D_{Y}\right)$ be a solution of (9) with $\mu>0$. Then

$$
0 \leq D_{x} \bullet D_{Y} \leq 2 \delta^{2} .
$$

In addition, the spectral norm of $D_{X Y}$ satisfies

$$
\left\|D_{X Y}\right\| \leq \frac{1}{4}\left\|P_{V}\right\|_{F}^{2}=\delta^{2} .
$$

Lemma 4 (Lemma 6.3.2 in [8]). One has $\left\|D_{X Y}\right\|_{F}^{2} \leq \frac{1}{8}\left\|P_{V}\right\|_{F}^{4}$.

Also we briefly recall some known facts from linear algebra for the trace of an invertible square matrix and its eigenvalues which will be used later.

Property 1. Let $A, B \in \mathbb{R}^{n \times n}$, then

1. $\operatorname{Tr}(A+B)=\operatorname{Tr}(A)+\operatorname{Tr}(B)$;

2. $\operatorname{Tr}(\beta A)=\beta \operatorname{Tr}(A)$;

3. $\operatorname{Tr}(A)=\operatorname{Tr}\left(A^{T}\right)$;

4. $\operatorname{Tr}(A)=\operatorname{Tr}(B)$ if $A \sim B$;

5. $\operatorname{Tr}(A B)=\operatorname{Tr}(B A)$;

6. $\operatorname{Tr}(A)=0$ if $A=-A^{T}$, i.e., A skew-symmetric;

7. $\lambda_{\max }\left(A^{2}\right)=\left(\lambda_{\max }(A)\right)^{2}$;

8. $\lambda_{\max }\left(A^{-1}\right)=\frac{1}{\lambda_{\min }(A)}$.

The next lemma gives a sufficient condition for strict feasibility of the full NT-step under the condition $\delta<1$.

Lemma 5. Let $\delta<1$, then the full NT-step is strictly feasible.

Proof. By applying (7) and (8), we have

$$
\begin{aligned}
X(\alpha) Y(\alpha) & =X Y+\alpha(\Delta X Y+X \Delta Y)+\alpha^{2} \Delta X \Delta Y \\
& =\mu D\left[V^{2}+\alpha\left(D_{X} V+V D_{Y}\right)+\alpha^{2} D_{X} D_{Y}\right] D^{-1} \\
& \sim \mu\left[V^{2}+\alpha\left(D_{X} V+V D_{Y}\right)+\alpha^{2} D_{X} D_{Y}\right] .
\end{aligned}
$$

Defining now the two matrices $M(\alpha)$ and $B(\alpha)$ as follows:

$$
\begin{aligned}
M(\alpha) & =\mu\left[\frac{1}{2} \alpha\left(D_{X} V+V D_{Y}-V D_{X}-D_{Y} V\right)+\frac{\alpha^{2}}{2}\left(D_{X} D_{Y}-D_{Y} D_{X}\right)\right] \\
B(\alpha) & =\mu\left[V^{2}+\frac{1}{2} \alpha\left(D_{X} V+V D_{Y}+V D_{X}+D_{Y} V\right)+\alpha^{2} D_{X Y}\right] \\
& =\mu\left[V^{2}+\frac{1}{2} \alpha\left(\left(D_{X}+D_{Y}\right) V+V\left(D_{Y}+D_{X}\right)\right)+\alpha^{2} D_{X Y}\right] \\
& =\mu\left[(1-\alpha) V^{2}+\alpha\left(I+\alpha D_{X Y}\right)\right] .
\end{aligned}
$$


According to (13), we have

$$
X(\alpha) Y(\alpha) \sim \mu\left[V^{2}+\alpha\left(D_{X} V+V D_{Y}\right)+\alpha^{2} D_{X} D_{Y}\right]=M(\alpha)+B(\alpha) .
$$

One can easily check that the matrix $M(\alpha)$ is skew-symmetric. Hence, Lemma 1 implies that $\operatorname{det}(X(\alpha) Y(\alpha))>0$, if the matrix $B(\alpha) \succ 0$. Then, from Lemma 2, $B(\alpha) \succ 0$ if $\alpha \leq 1$ and $\left\|D_{X Y}\right\|<1$. By Lemma 4, $\left\|D_{X Y}\right\| \leq \frac{1}{4}\left\|P_{V}\right\|_{F}^{2}=\delta^{2}<1$. In addition, since $X(0) \succ 0$ and $Y(0) \succ 0$, Lemma 1, implies that $X(1) \succ 0$ and $Y(1) \succ 0$. This completes the proof of the lemma.

Finally, we may write

$$
V_{+}^{2}=\frac{1}{\mu} D^{-1} X_{+} Y_{+} D=\frac{1}{\mu} D^{-1} X(1) Y(1) D
$$

This implies that

$$
\mu V_{+}^{2} \sim X_{+} Y_{+} \sim X(1) Y(1)
$$

Lemma 6. One has

$$
\lambda_{\min }\left(V_{+}^{2}\right) \geq 1-\delta^{2}
$$

Proof. In view of (14) and (15) in the proof of the Lemma 5, letting $\alpha=1$, we get

$$
X_{+} Y_{+}=X(1) Y(1) \sim B(1)+M(1) \sim \mu I+\mu D_{X Y}+M(1)
$$

Due to (16), it follows that

$$
V_{+}^{2} \sim I+D_{X Y}+\frac{1}{\mu} M(1)
$$

and consequently we have,

$$
\lambda_{\min }\left(V_{+}^{2}\right)=\lambda_{\min }\left(I+D_{X Y}+\frac{1}{\mu} M(1)\right) .
$$

On the other hand, by Lemma 2, we derive

$$
\lambda_{\min }\left(V_{+}^{2}\right) \geq \lambda_{\min }\left(I+D_{X Y}\right) \geq 1-\left|\lambda_{\min }\left(D_{X Y}\right)\right| \geq 1-\left|\lambda_{\max }\left(D_{X Y}\right)\right|=1-\left\|D_{X Y}\right\| .
$$

Lemma 3, (12) implies that

$$
\lambda_{\min }\left(V_{+}^{2}\right) \geq\left(1-\frac{1}{4}\left\|P_{V}\right\|_{F}^{2}\right) \geq 1-\delta^{2} .
$$

This completes the proof.

Lemma 7. If $\delta<1$. Then

$$
\delta_{+}:=\delta\left(X_{+}, Y_{+} ; \mu\right) \leq \frac{\delta^{2}}{\sqrt{2\left(1-\delta^{2}\right)}} .
$$

Moreover, If $\delta \leq \frac{2}{\sqrt{10}}$, then $\delta_{+} \leq \delta^{2}$ which shows the quadratic convergence of Algorithm 2.3.

Proof. We have,

$$
\delta^{2}\left(X_{+}, Y_{+} ; \mu\right)=\frac{1}{4}\left\|V_{+}^{-1}-V_{+}\right\|_{F}^{2}=\frac{1}{4}\left\|V_{+}^{-1}\left(I-V_{+}^{2}\right)\right\|_{F}^{2} \leq \frac{1}{4} \lambda_{\max }^{2}\left(V_{+}^{-1}\right)\left\|I-V_{+}^{2}\right\|_{F}^{2} .
$$


Hence,

$$
\delta^{2}\left(X_{+}, Y_{+} ; \mu\right) \leq \frac{1}{4} \frac{1}{\lambda_{\min }\left(V_{+}^{2}\right)}\left\|I-V_{+}^{2}\right\|_{F}^{2} .
$$

By Lemma 6, it follows that

$$
\delta^{2}\left(X_{+}, Y_{+} ; \mu\right) \leq \frac{1}{4\left(1-\delta^{2}\right)}\left\|I-V_{+}^{2}\right\|_{F}^{2}
$$

Next task, is to show that

$$
\left\|I-V_{+}^{2}\right\|_{F}^{2} \leq\left\|D_{X Y}\right\|_{F}^{2}
$$

From (17), we deduce that

$$
I-V_{+}^{2} \sim-D_{X Y}-\frac{1}{\mu} M(1)
$$

and so

$$
\left\|I-V_{+}^{2}\right\|_{F}=\left\|D_{X Y}+\frac{1}{\mu} M(1)\right\|_{F} .
$$

We have,

$$
\begin{aligned}
\left\|I-V_{+}^{2}\right\|_{F}^{2} & =\operatorname{Tr}\left(\left(D_{X Y}+\frac{1}{\mu} M(1)\right)^{2}\right) \\
& =\operatorname{Tr}\left(D_{X Y}^{2}+\frac{1}{\mu^{2}} M^{2}(1)\right)+\frac{2}{\mu} \operatorname{Tr}\left(D_{X Y} M(1)\right)
\end{aligned}
$$

Next, we show that

$$
\frac{2}{\mu} \operatorname{Tr}\left(D_{X Y} M(1)\right)=0 .
$$

Since $M$ is skew-symmetric and $D_{X Y}$ is symmetric, we have,

$$
\begin{aligned}
\operatorname{Tr}\left(D_{X Y} M(1)\right) & =-\operatorname{Tr}\left(D_{X Y}^{T} M^{T}(1)\right)=-\operatorname{Tr}\left(\left(M(1) D_{X Y}\right)^{T}\right) \\
& =-\operatorname{Tr}\left(M(1) D_{X Y}\right)=-\operatorname{Tr}\left(D_{X Y} M(1)\right) .
\end{aligned}
$$

This implies that

$$
\operatorname{Tr}\left(D_{X Y} M(1)\right)=0 .
$$

Hence, we get

$$
\begin{aligned}
\left\|I-V_{+}^{2}\right\|_{F}^{2} & =\operatorname{Tr}\left(D_{X Y}^{2}-\frac{1}{\mu^{2}} M(1) M(1)^{T}\right) \\
& =\left\|D_{X Y}\right\|_{F}^{2}-\frac{1}{\mu^{2}}\|M(1)\|_{F}^{2} \leq\left\|D_{X Y}\right\|_{F}^{2} .
\end{aligned}
$$

Finally, it follows that $\left\|I-V_{+}\right\|_{F} \leq\left\|D_{X Y}\right\|$. By Lemma 4, the result followed. This completes the proof.

The next lemma gives the effect of the full NT-step on duality gap.

Lemma 8. Let $\delta \leq \frac{2}{\sqrt{10}}$. After a full NT-step one has $X_{+} \bullet Y_{+} \leq 2 \mu n$. 
Proof. Due to (16), $V_{+}^{2} \sim \frac{1}{\mu} X_{+} Y_{+}$and the fact that $\operatorname{Tr}(M(1))=0$, and using (17) we obtain

$$
\begin{aligned}
X_{+} \bullet Y_{+} & =\mu \operatorname{Tr}\left(V_{+}^{2}\right)=\mu \operatorname{Tr}\left(I+D_{X Y}+M(1)\right) \\
& =\mu \operatorname{Tr}\left(I+D_{X Y}\right)=\mu\left(n+\operatorname{Tr}\left(D_{X Y}\right)\right) \\
& =\mu\left(n+\operatorname{Tr}\left(D_{X} D_{Y}\right)\right)=\mu\left(n+D_{X} \bullet D_{Y}\right) .
\end{aligned}
$$

Now due to (11), it follows

$$
X_{+} \bullet Y_{+} \leq \mu\left(n+2 \delta^{2}\right)
$$

Let $\delta \leq \frac{2}{\sqrt{10}}$, then

$$
X_{+} \bullet Y_{+} \leq \mu\left(n+\frac{4}{5}\right) .
$$

Because $\left(n+\frac{4}{5}\right) \leq 2 n$ for all $n \geq 1$, the result followed. This completes the proof.

\subsection{Updating the barrier parameter}

In the following subsection, we investigate the effect on the proximity measure of a full NT-step by an update of the parameter $\mu$.

Lemma 9. If $\delta \leq \frac{2}{\sqrt{10}}$ and $\mu_{+}=(1-\theta) \mu$, where $0 \leq \theta \leq 1$, then

$$
\delta^{2}\left(X_{+}, Y_{+} ; \mu_{+}\right) \leq \frac{2}{15}+\frac{\theta^{2}}{4(1-\theta)}\left(n+\frac{4}{5}\right)+\frac{4 \theta}{15} .
$$

Moreover, if $\delta \leq \frac{2}{\sqrt{10}}, \theta=\left(\frac{6}{23 n}\right)^{1 / 2}$ and $n \geq 2$, then $\delta\left(X_{+}, Y_{+} ; \mu_{+}\right) \leq \frac{2}{\sqrt{10}}$.

Proof. We have,

$$
\begin{aligned}
4 \delta^{2}\left(X_{+}, Y_{+} ; \mu_{+}\right) & =\left\|\sqrt{(1-\theta)} V_{+}^{-1}-\frac{V_{+}}{\sqrt{(1-\theta)}}\right\|_{F}^{2} \\
& =\left\|\sqrt{1-\theta}\left(V_{+}^{-1}-V_{+}\right)-\frac{\theta}{\sqrt{(1-\theta)}} V_{+}\right\|_{F}^{2} \\
& =(1-\theta)\left\|V_{+}^{-1}-V_{+}\right\|_{F}^{2}+\frac{\theta^{2}}{1-\theta}\left\|V_{+}\right\|_{F}^{2}-2 \theta \operatorname{Tr}\left(\left(V_{+}^{-1}-V_{+}\right) V_{+}\right) \\
& =(1-\theta)\left\|V_{+}^{-1}-V_{+}\right\|_{F}^{2}+\frac{\theta^{2}}{1-\theta}\left\|V_{+}\right\|_{F}^{2}-2 \theta \operatorname{Tr}\left(I-V_{+}^{2}\right) \\
& =4(1-\theta) \delta_{+}^{2}+\frac{\theta^{2}}{1-\theta}\left\|V_{+}\right\|_{F}^{2}-2 \theta n+2 \theta\left\|V_{+}\right\|_{F}^{2} .
\end{aligned}
$$

Let $V_{+}^{2}=\frac{1}{\mu} D^{-1} X_{+} Y_{+} D$ and due to (18) in the proof of Lemma 8, we have

$$
\left\|V_{+}\right\|_{F}^{2}=\operatorname{Tr}\left(V_{+}^{2}\right)=\frac{1}{\mu} X_{+} \bullet Y_{+} \leq\left(n+\frac{4}{5}\right) .
$$

Hence, after some elementary reductions, we obtain

$$
\delta^{2}\left(X_{+}, Y_{+} ; \mu_{+}\right) \leq(1-\theta) \delta_{+}^{2}+\frac{\theta^{2}}{4(1-\theta)}\left(n+\frac{4}{5}\right)+\frac{2 \theta}{5} .
$$


By Lemma $7, \delta_{+} \leq \frac{\delta^{2}}{\sqrt{2\left(1-\delta^{2}\right)}}$ and if $\delta \leq \frac{2}{\sqrt{10}}$, then we get

$$
\delta^{2}\left(X_{+}, Y_{+} ; \mu_{+}\right) \leq \frac{2}{15}+\frac{\theta^{2}}{4(1-\theta)}\left(n+\frac{4}{5}\right)+\frac{4 \theta}{15} .
$$

Let $\theta=\left(\frac{6}{23 n}\right)^{1 / 2}$ then $\theta^{2}=\frac{6}{23 n}$, and we have,

$$
\delta^{2}\left(X_{+}, Y_{+} ; \mu_{+}\right) \leq \frac{2}{15}+\frac{\frac{6}{23 n}\left(n+\frac{4}{5}\right)}{4(1-\theta)}+\frac{4 \theta}{15} .
$$

Because $\frac{6\left(n+\frac{4}{5}\right)}{23 n} \leq \frac{42}{115}$ for all $n \geq 2$, this implies that,

$$
\delta^{2}\left(X_{+}, Y_{+} ; \mu_{+}\right) \leq f(\theta)=\frac{2}{15}+\frac{21}{230(1-\theta)}+\frac{4 \theta}{15} .
$$

Also for $n \geq 2$, then $0 \leq \theta \leq\left(\frac{3}{23}\right)^{1 / 2}$. Since

$$
f^{\prime}(\theta)=\frac{21}{230(1-\theta)^{2}}+\frac{4}{15}>0, \text { for all } 0<\theta<\left(\frac{3}{23}\right)^{1 / 2},
$$

then $f(\theta)$ is continuous and monotonic increasing function on $\left[0,\left(\frac{3}{23}\right)^{1 / 2}\right]$, so

$$
f(\theta) \leq f\left(\left(\frac{3}{23}\right)^{1 / 2}\right)=0.3725<\frac{4}{10} .
$$

Hence $\delta\left(X_{+}, Y_{+} ; \mu_{+}\right) \leq \frac{2}{\sqrt{10}}$. This completes the proof.

Lemma 9, indicates that for the defaults $\tau=\frac{2}{\sqrt{10}}$ and $\theta=\left(\frac{6}{23 n}\right)^{1 / 2}$, the Algorithm 2.3, is well-defined since the conditions $X_{+} \succ 0, Y_{+} \succ 0$ and $\delta\left(X_{+}, Y_{+} ; \mu_{+}\right) \leq \frac{2}{\sqrt{10}}$ are maintained during the solution process.

\subsection{Iteration bound}

We conclude this section with a theorem that gives us the iteration bound of Algorithm 2.3. Before doing this we apply the results obtained in the previous subsections and get the following lemma.

Lemma 10. Assume that $X^{0}$ and $Y^{0}$ are strictly feasible starting point for $S D L C P$ (1) such that $\delta\left(X^{0}, Y^{0} ; \mu_{0}\right) \leq \frac{2}{\sqrt{10}}$ for certain $\mu_{0}>0$. Moreover, let $X^{k}$ and $Y^{k}$ be the iterate produced by Algorithm 2.3, after $k$ iterations. Then, the inequality $X^{k} \bullet Y^{k} \leq \epsilon$ is satisfied for

$$
k \geq \frac{1}{\theta} \log \left(\frac{2 n \mu_{0}}{\epsilon}\right) .
$$

Proof. After $k$ iterations, Lemma 8 implies that

$$
X^{k} \bullet Y^{k} \leq 2 n \mu_{k}=2 n(1-\theta)^{k} \mu_{0} .
$$

Then $X^{k} \bullet Y^{k} \leq \epsilon$ holds if $2 n(1-\theta)^{k} \mu_{0} \leq \epsilon$. Taking logarithm, we arrive at the relation

$$
k \log (1-\theta) \leq \log \epsilon-\log \left(2 n \mu_{0}\right) .
$$

Using $-\log (1-\theta) \geq \theta$, for $0 \leq \theta \leq 1$, then, we deduce that the above inequality holds if

$$
k \theta \geq \log 2 n \mu_{0}-\log \epsilon=\log \left(\frac{2 n \mu_{0}}{\epsilon}\right) .
$$

This implies the lemma. 
Theorem 1. Let $\theta=\left(\frac{6}{23 n}\right)^{1 / 2}$ and $\tau=\frac{2}{\sqrt{10}}$ with $\mu_{0}=\frac{1}{2}$. Then, Algorithm 2.3 requires at most

$$
O\left(\sqrt{n} \log \frac{n}{\epsilon}\right)
$$

iterations for getting an $\epsilon$-approximate solution of SDLCP.

\section{Numerical results}

In this section, we shall test Algorithm 2.3 on two monotone SDLCPs to validate its efficiency. To achieve this, we provide a feasible starting point $\left(X^{0}, Y^{0}\right)$ such that IPC and $\delta\left(X^{0}, Y^{0} ; \mu_{0}\right) \leq$ $\frac{2}{\sqrt{10}}$ are satisfied. We have implemented Algoritm 2.3 in MATLAB and run on Pentium 4. In the implementation, we use $\epsilon=10^{-6}, \theta=\left(\frac{6}{23 n}\right)^{1 / 2}$ and $\tau=\frac{2}{\sqrt{10}}$. Also we make change in the theoretical barrier parameter $\mu_{0}>0$ where some relaxed barrier values are suggested in order to reduce the number of iterations produced by the algorithm. The number of iterations and the time produced by Algorithm 2.3, are denoted by "Iter " and "CPU", respectively. We note here that the first example of monotone SDLCP is reformulated from the symmetric semidefinite constrained least squares (SDLS) problem [14]. However, the second example is defined by the double sided multiplicative linear transformation. Finally, we compare our obtained numerical results with those obtained by using the classical defaults $\theta=\frac{1}{2 \sqrt{n}}$ and $\tau=\frac{1}{2}$.

\section{Problem 1.}

The symmetric semidefinite constrained least squares problem (SDLS) is defined as the following convex optimization problem:

$$
\min _{X} \frac{1}{2}\|A X-B\|_{F}^{2} \text { s.t. } X \succeq 0
$$

where $A, B$ are given matrices in $\mathbb{R}^{m \times n}$ with $m \geq n$. It is shown that if $\operatorname{rank}(A)=n$, then SDLS has a unique solution $X^{*} \succeq 0$ [14]. Therefore, the necessary and sufficient optimality conditions for SDLS are equivalent to the following monotone SDLCP where $\mathcal{L}(X)$ and $Q$ are given by:

$$
\mathcal{L}(X)=\frac{1}{2}\left(A^{T} A X+X A^{T} A\right) \text { and } Q=-\frac{1}{2}\left(A^{T} B+B^{T} A\right) .
$$

Here $\mathcal{L}(X)$ is named as the Lyapunov linear transformation and it is easy to check that $\mathcal{L}(X)$ is symmetric and strictly monotone for all $X$ in $\mathbb{S}^{n}[9]$. For example, for $m=6$ and $n=5$, the matrices $A$ and $B$ of the SDLS are given by:

$$
\begin{aligned}
A & =\left(\begin{array}{ccccc}
6 & -1 & 0 & 0 & 0 \\
-0.1 & 6 & -1 & 0 & 0 \\
0 & -0.1 & 6 & -1 & 0 \\
0 & 0 & -0.1 & 6 & -1 \\
0 & 0 & 0 & -0.1 & 6 \\
0 & 0 & 0 & 0 & -0.1
\end{array}\right), \\
B & =\left(\begin{array}{ccccc}
1 & 0 & 0 & 0 & 0 \\
-0.4 & 1 & 0 & 0 & 0 \\
-0.4 & -0.4 & 1 & 0 & 0 \\
-0.4 & 0 & -0.4 & 1 & 0 \\
-0.4 & 0 & 0 & -0.4 & 1 \\
-0.4 & 0 & 0 & 0 & -0.4
\end{array}\right) .
\end{aligned}
$$

The unique optimal solution $X^{*} \in \mathbb{S}_{+}^{5}$ is given by: 


$$
X^{*}=\left(\begin{array}{ccccc}
0.1639 & -0.0215 & -0.0342 & -0.0328 & -0.0300 \\
-0.0215 & 0.1553 & -0.0227 & -0.0019 & -0.0027 \\
-0.0342 & -0.0227 & 0.1558 & -0.0194 & 0.0014 \\
-0.0328 & -0.0019 & -0.0194 & 0.1564 & -0.0189 \\
-0.0300 & -0.0027 & 0.0014 & -0.0189 & 0.1598
\end{array}\right)
$$

The numerical results obtained by the application of Algorithm 2.3, to the corresponding SDLCP in (19), with the strictly feasible initial starting point $X^{0} \succ 0$ is given by:

$$
X^{0}=\operatorname{Diag}(0.2369, \ldots, 0.2369)
$$

\begin{tabular}{|c|c|c|c|c|}
\hline$\theta / \mu$ & 0.5 & 0.05 & 0.005 & 0.0005 \\
\hline & Iter $\mathrm{CP}$ & Iter $\mathrm{CPU}$ & $\begin{array}{ll}\text { Iter } & \mathrm{CPU}\end{array}$ & Iter $\mathrm{CPU}$ \\
\hline$\left(\frac{6}{23}\right)^{1}$ & 0.03 & $42 \quad 0.0235$ & $\begin{array}{ll}33 & 0.0230\end{array}$ & $24 \quad 0.0206$ \\
\hline$\frac{1}{2 \sqrt{n}}$ & $\begin{array}{ll}55 & 0.036\end{array}$ & 450.0323 & $\begin{array}{ll}34 & 0.0321\end{array}$ & $\begin{array}{ll}25 & 0.0291\end{array}$ \\
\hline
\end{tabular}

are summarized in table 1.

Table 1: Numerical results for Problem 1.

Problem 2.

The data of the monotone SDLCP is given by:

$$
\mathcal{L}(X)=A X A^{T}+Q,
$$

where

$$
A=\left(\begin{array}{ccccc}
17.25 & -1.75 & -1.75 & -1.75 & -1.75 \\
-1.75 & 16.25 & -2 & 0 & 0 \\
-1.75 & -2 & 16.25 & -2 & 0 \\
-1.75 & 0 & -2 & 16.25 & -2 \\
-1.75 & 0 & 0 & -2 & 16.25
\end{array}\right)
$$

and

$$
Q=\left(\begin{array}{ccccc}
-9.25 & 1.25 & 1.25 & 1.25 & 1.25 \\
1.25 & -8.25 & 1.5 & 0 & 0 \\
1.25 & 1.5 & -8.25 & 1.5 & 0 \\
1.25 & 0 & 1.5 & -8.25 & 1.5 \\
1.25 & 0 & 0 & 1.5 & -8.25
\end{array}\right)
$$

Note here since the matrix $A$ is positive definite, it has shown [10], that the symmetric double sided multiplicative linear transformation is strictly monotone and so the corresponding SDLCP in (20), has a unique solution for all symmetric $Q$. The unique solution $X^{*} \in \mathbb{S}_{+}^{5}$ of the proposed example is given by:

$$
X^{*}=\left(\begin{array}{ccccc}
0.0313 & 0.0020 & 0.0020 & 0.0020 & 0.0020 \\
0.0020 & 0.0313 & 0.0019 & 0 & 0 \\
0.0020 & 0.0019 & 0.0312 & 0.0019 & 0 \\
0.0020 & 0 & 0.0019 & 0.0312 & 0.0019 \\
0.0020 & 0 & 0 & 0.0019 & 0.0313
\end{array}\right)
$$

The numerical results obtained by Algorithm 2.3, for this problem with the strictly feasible initial starting point $X^{0} \succ 0$ is given by:

$$
X^{0}=\operatorname{Diag}(0.0620, \ldots, 0.0620),
$$


are summarized in table 2.

\begin{tabular}{|l|ll|ll|ll|ll|}
\hline$\theta / \mu$ & \multicolumn{2}{|l|}{0.5} & & 0.05 & \multicolumn{2}{|l|}{0.005} & \multicolumn{2}{|l|}{0.0005} \\
\hline & Iter & CPU & Iter & CPU & Iter & CPU & Iter & CPU \\
\hline$\left(\frac{6}{23}\right)^{1 / 2}$ & 51 & 0.0184 & 42 & 0.0213 & 33 & 0.0182 & 24 & 0.0163 \\
\hline$\frac{1}{2 \sqrt{n}}$ & 53 & 0.0186 & 43 & 0.0227 & 35 & 0.0235 & 25 & 0.0202 \\
\hline
\end{tabular}

Table 2: Numerical results for Problem 2.

It can be observed from Tables 1 and 2 that the numbers of iteration and the time produced by our algorithm with these new defaults are better than those obtained by the most used defaults.

\section{Conclusion and future works}

In this paper, we have extended a primal-dual path-following interior-point algorithm for SDO and CQSDO to monotone SDLCPs. The corresponding algorithm is based only on full NesterovTodd step and on new defaults. We derived the currently best known iteration bound for the algorithm with short-step method, namely, $O\left(\sqrt{n} \log \frac{n}{\epsilon}\right)$ which is the same as in the LO, SDO and CQSDO cases. The practical performance of the algorithm is demonstrated by its success to solve some monotone SDLCPs linked to some well-known linear transformations such as Lyapunov and two-sided. Further research might extend the algorithm to linear complementarity problems over symmetric cones.

\section{References}

[1] Achache., M. (2006). A new primal-dual path-following method for convex quadratic programming. Computational and Applied Mathematics, 25(1), 97-110.

[2] Achache, M. and Guerra, L. (2014). A full Nesterov-Todd-step feasible primal-dual interior-point algorithm for convex quadratic semi-definite optimization. Applied Mathematics and Computation, 231, 581-590.

[3] Achache, M. and Boudiaf, N. (2011). Complexity analysis of primal-dual algorithms for semidefinite linear complementarity problems. Revue d'Analyse Numérique et de Théorie de l'Approximation, 40 (2) 2, 95-106.

[4] Achache, M., Boudiaf, N. and A. (2009). Keraghel. Le problème de complémentarité linéaire semi-défini. Revue d'Analyse Numérique et de Théorie de l'Approximation, 38 (2), 115-129.

[5] Achache, M. (2010). Complexity analysis and numerical implementation of a short-step primaldual algorithm for linear complementarity problems. Applied Mathematics and Computation, 216 (7), 1889-1895.

[6] Chen, F. (2011). Polynomial convergence of primal-dual algorithms for SDLCP based on the M-Z family of directions. Applied Mathematical Sciences, 5 (39), 1903-1907.

[7] Darvay, Zs. (2003). New interior-point algorithms for linear optimization. Advanced Modeling Optimization, 5 (1), 51-92.

[8] De Klerk, E. (1997). Interior point methods for semidefinite programming. Master of Science in the Faculty of Engineering. University of Pretoria.

[9] Gowda, M. S. and Song, Y. (2000). On semidefinite linear complementarity problem. Mathematical Programming, Series A, 88, 575-587.

[10] Gowda M. S., Song, Y. and Ravindran, G. (2003). On some interconnections between strict monotonicity, globally uniquely solvable, and $\mathrm{P}$ properties in semidefinite linear complementarity problems. Linear Algebras and its Applications, 370, 355-368.

[11] Kheirfam, B. (2013). A new infeasible interior-point algorithm with full Nesterov-Todd step for semidefinite Optimization. Iranian Journal of Operations Research, 4(1), 88-107.

[12] Kheirfam, B. and Haghighi, M. (2016). A full-Newton step feasible interior-point algorithm for $P_{*}(\kappa)$-LCP based on a new search direction. Croatian Operational Research Review, 7, 277-290. 
[13] Kojima, M., Shindoh, M. and Hara, S. (1997). Interior-point methods for the monotone semidefinite linear complementarity in symmetric matrices. SIAM J. Optimization, 7, 86-125.

[14] Krislock, N. G. B. (2003). Numerical solution of semidefinite constrained least squares problems. Master of Science. The university of British Colombia, Canada.

[15] Mansouri, H., Zangiabadi, M. and Pirhaji, M. (2016). A polynmial-time interior-point algorithm for linear semidefinite complementarity problems. Mathematical Analysis and Optimization, 1(1), $37-45$.

[16] Nesterov, Y. E. and Todd, M. J. (1997). Self-scaled barriers and interior-point methods for convex programming. Mathematics of Operations Research, 22(1), 1-42.

[17] Roos, C., Terlaky, T. and Vial, Ph. J. (1997). Theory and Algorithms for linear optimization. An Interior Point Approach. John Wiley and Sons, Chichester, UK.

[18] Tseng, P. (1998). Search directions and convergence analysis of some infeasible path-following methods for the monotone semi-definite LCP. Journal of Optimization Methods and Software, 59, 245-268.

[19] Wang, G. Q. and Bai, Y. Q. (2009). A new primal-dual path-following interior point algorithm for semidefinte optimization. Journal of Mathematical Analysis and Applications, 353, 339-349. 